\title{
CORRIGENDUM
}

\section{Evidence of association between bipolar disorder and Citron on chromosome 12q24}

A Lyons-Warren ${ }^{1}$, JJ Chang ${ }^{1}, \mathrm{R}$ Balkissoon ${ }^{1}$, A Kamiya $^{1}$, M Garant $^{2}$, J Nurnberger ${ }^{3}$, W Scheftner T Reich $^{5}$, FJ McMahon ${ }^{6}$, J Kelsoe ${ }^{7,8}$, E Gershon ${ }^{9}$, W Coryell ${ }^{10}$, W Byerley ${ }^{11}$, W Berrettini ${ }^{12}$, R DePaulo ${ }^{1}$, M Mclnnis ${ }^{1,13}$ and A Sawa ${ }^{1,14}$

${ }^{1}$ Department of Psychiatry, Johns Hopkins University School of Medicine, Baltimore, MD, USA; ${ }^{2}$ Division of Endocrinology, Diabetes and Nutrition, University of Maryland School of Medicine, Baltimore, MD, USA; ${ }^{3}$ Indiana University, Indianapolis, IN, USA; ${ }^{4}$ Rush-Presbyterian Medical Center, St Louis, MO, USA; ${ }^{5}$ Washington University, St Louis, MO, USA; ${ }^{6}$ Genetic Basis of Mood and Anxiety Disorders, National Institute of Mental Health, National institutes of Health, US Department of Health and Human Services, Bethesda, MD, USA; ${ }^{7}$ University of California San Diego, San Diego, CA, USA; ${ }^{8}$ San Diego Veterans Affairs Healthcare System, San Diego, CA, USA; ${ }^{9}$ University of Chicago, Chicago, IL, USA; ${ }^{10}$ University of Iowa, Iowa City, IA, USA; ${ }^{11}$ University of California Irvine, Irvine, CA, USA; ${ }^{2}$ University of Pennsylvania, Philadelphia, PA, USA; ${ }^{13}$ Department of Psychiatry, University of Michigan, Ann Arbor, MI, USA and ${ }^{14}$ Department of Neuroscience, Johns Hopkins University School of Medicine, Baltimore, MD, USA

Molecular Psychiatry (2006) 11, 612. doi:10.1038/sj.mp.4001842

Correction to: Molecular Psychiatry (2005) 10, 807-809. doi: 10.1038/sj.mp.4001703

Following publication of the above article, the authors identified an algorithmic error in the analysis. Table 1 below reflects a corrected analysis of the data set, and shows a positive association within a haplo- block containing SNPs rs278124 and rs278109, in affected offspring. Minor changes to the table were also incorporated for clarity. Although we may not have evidence that genetic variations in Citron influence the protein interaction of Citron and DISC1, the data nevertheless suggests a possible association between Citron and bipolar disorder.

Table 1 FBAT results for Citron; 12q24.23

\begin{tabular}{|c|c|c|c|c|c|c|c|c|c|c|c|c|c|c|c|c|}
\hline $\begin{array}{l}L D \\
d b S N P-I D\end{array}$ & $\begin{array}{l}\text { CELERA } \\
I D\end{array}$ & Location $^{\mathrm{a}}$ & $S N P^{b}$ & $M A^{\mathrm{c}}$ & Frequency & $\begin{array}{l}\text { obs/ } \\
\text { exp }\end{array}$ & $\begin{array}{c}P- \\
\text { value }\end{array}$ & \multicolumn{3}{|c|}{$\begin{array}{l}\text { Over-transmitted } \\
\text { to affected } \\
\text { offspring }\end{array}$} & \multicolumn{6}{|c|}{$\begin{array}{l}\text { Under-transmitted } \\
\text { to affected } \\
\text { offsping }\end{array}$} \\
\hline $\begin{array}{l}\text { rs } 278124 \\
\text { rs } 2285595 \\
\text { rs } 278109 \\
\text { rs } 2074052 \\
\text { rs } 203368 \\
\text { rs } 435136\end{array}$ & $\begin{array}{l}\text { hCV1078831 } \\
\text { hCV3259859 } \\
\text { hCV1078854 } \\
\text { hCV2547173 } \\
\text { hCV2626794 } \\
\text { hCV2626820 } \\
\text { hCV3259834 }\end{array}$ & $\begin{array}{l}\text { Intron46 } \\
\text { Intron31 } \\
\text { Exon26-R/R } \\
\text { Intron21 } \\
\text { Intron13 } \\
\text { Intron9 } \\
\text { Intron5 } \\
\text { q-telomere }\end{array}$ & $\begin{array}{l}\text { T/C } \\
\text { T/C } \\
\text { G/A } \\
\text { G/C } \\
\text { G/A } \\
\text { C/A } \\
\text { G/A }\end{array}$ & $\begin{array}{l}\mathrm{C} \\
\mathrm{C} \\
\mathrm{A} \\
\mathrm{C} \\
\mathrm{A} \\
\mathrm{T} \\
\mathrm{A}\end{array}$ & $\begin{array}{l}0.160 \\
0.460 \\
0.182 \\
0.467 \\
0.451 \\
0.408 \\
0.492\end{array}$ & $\begin{array}{l}88 / 103 \\
96 / 113\end{array}$ & $\begin{array}{l}0.016 \\
0.009 \\
\end{array}$ & $\begin{array}{l}\mathbf{0 . 0 3 7} \\
0.56\end{array}$ & $\begin{array}{l}\mathrm{T} \\
\mathrm{T} \\
\mathrm{G}\end{array}$ & $\begin{array}{l}\mathbf{0 . 0 5 0} \\
0.44\end{array}$ & $\begin{array}{l}\mathbf{0 . 0 1 0} \\
0.13\end{array}$ & $\begin{array}{l}\mathbf{0 . 0 0 5} \\
0.16\end{array}$ & $\begin{array}{l}\mathbf{0 . 0 1 8} \\
0.16\end{array}$ & $\begin{array}{l}\mathbf{0 . 0 0 7} \\
0.11\end{array}$ & $\begin{array}{l}\mathbf{0 . 0 1 8} \\
0.16\end{array}$ & $\begin{array}{l}\text { C } \\
\text { C } \\
\text { A } \\
\text { G }\end{array}$ \\
\hline
\end{tabular}

$\mathrm{D}^{\prime}>0.95$.

aRelative to GenBank AY681966.

${ }^{\mathrm{b}}$ On human citron coding strand.

${ }^{\mathrm{c}}$ Minor allele.

Bold type indicates $P<0.05$. 